ALIMTA $^{\oplus} 100 \mathrm{mg}$ bzw. $500 \mathrm{mg}$ Pulver zur Herstellung eines Konzentrates zur Herstellung einer Infusionslösung. Wirkstoff: Pemetrexed. Zusammensetzung: Jede Durchstechflasche enthält $100 \mathrm{mg}$ bzw. $500 \mathrm{mg}$ Pemetrexed (als Pemetrexeddinatrium). Nach Auflösung enth Pemetrexeddinatric $5 \mathrm{mg} / \mathrm{ml}$ Pemetrexed. Sonstige Bestandteile: Mannitol, Salzsäure, Natriumhydroxid (enthält ca. $11 \mathrm{mg}$ bzw. $54 \mathrm{mg}$ Natrium). Anwendungsgebiete: Malignes Pleuramesotheliom: In Kombination mit Cisplatin zur Behandlung von chemonaiven Patienten mit inoperabem malignen Pleuramesotheliom. Nichtkeinzelliges Lungenkarzinom: Zur first-Ine Therapie von Patienten mit lokal fortgeschrittenem oder metastasiertem nicht-kleinzelligen Lungenkarzinom außer bei überwiegender plattenepithelialer Histologie. In Monotherapie für die Erhaltungstherapie bei lokal fortgeschrittenem ode metastasiertem nicht-kleinzelligen Lungenkarzinom außer bei ubewiegender platten Lunthelaler Histoogie bei Pattenten, dere platinbasierten Chemotherapie nicht unmittelba fortgeschritten ist. In Monotherapie zur Behandlung in Zweitlinientherapie von Patienten mit lokal fortgeschrittenem oder metastasiertem nichtkleinzelligen Lungenkarzinom außer bei überwiegender plattenepithelialer Histologie. Gegenanzeigen: plattenepithela oder einen der sonstigen Bestandteile, Stillen, gleich zeitige Gelbfieberimpfung. Nebenwirkungen: Sehr häufig: Neutrophile/Granulozyten, Leukozyten, Hämoglobin, Thrombozyten erniedrigt, Diarrhoe, Erbrechen, Stomatitis/Pharyngitis, Übelkeit, Appetitverlust, Obstipation, Müdigkeit, Neuropathie (Empfindungsstörung), Kreatinin Clearance erniedrigt, Hautrötung/Abschuppung, Haarausfall. Häufig: Erkrankungen der Augenoberfläche (einschl. Konjuktivitis), verstärkter Tränenfluss, Dyspepsie/Sodbrennen, Mukositis, Dehydratation, Geschmacksstörung, Infektion Sepsis (manchmal tödlich verlaufend), Fieber, febrile Neutropenie, AST (SGOT), Gamma-GT erhöht, ALT (SGPT) erhöht/erniedrigt, Nessesuc allergische Reaktionen/Überempfindlichkeitsreakt ionen, Brustschmerzen, motorische Neuropathie, sensorische Neuropathie, multiformes Erythem, Bauchschmerzen, Ödeme, verminderte glomeruläre Filtrationsrate, Nierenversagen, Schmerzen. Gelegentlich: Arrhythmie (u a supraventrikuläre) schwerwiegende kardiovaskulare und zerebrovasschiur Angina pectoris, zerebrovaskulärer Insult und transitorische ischämische Attacken (die meisten diese Patienten, hatten vorbestehende kardiovaskuläre Risikofaktoren), Panzytopenie, Colitis (einschließlich intestinale und rektale Blutungen, manchma tödlich verlaufende, intestinale Perforation, intestinale Nekrose und Typhlitis), manchmal tödlich verlaufende interstitielle Pneumonitis mit respiratorischer Insuffizienz, Ösophagitis/StrahlenÖsophagitis, Strahlenpneumonitis (bei Patienten, die vor, während oder nach ihrer Pemetrexed Therapie bestrahlt wurden), periphere Ischämien (die manchmal zu Nekrosen an den Extremitäten führen), Lungenembolie, Selten: Hepatitis, möglicherweise schwerwiegend, Radiation Recall" (bei Patienten, die vorher eine Strahlentherapie erhalten hatten), bullöse Erkrankungen einschließlich Stevens-Johnson Syndrom und toxischer epidermale Nekrolyse (in einigen Fällen tödlich verlaufend), hämolytische Anämie. Warnhinweise: Vorbehandlung und Begleittherapie mit Folsäure Vond Vitamin dig, weitere Warnhinweise s. Fachinformation. Verschreibungspflichtig. Pharm. Unternehmer Eli Lilly Nederland B.V., Grootslag 1-5, 3991 RA Houten, Niederlande. Vertrieb: Lilly Deutschlan GmbH, Werner-Reimers-Straße 2-4, 61352 Bad Homburg. Deutschland Stand der Information: Januar 2012 DEALM00932

\section{Wechselwirkungen unterschätzt Ältere Krebspatienten setzen unbedacht auf Knoblauch}

Alternativmedizinische Präparate wie etwa Knoblauchpillen können die Chemotherapiewirksamkeit schwächen. Für ältere Krebspatienten, die oft mehrere rezeptpflichtige Arzneien einnehmen, könnten solche Interaktionen besonders relevant sein. Eine Studie ergab nun, dass $17 \%$ der insgesamt 545 untersuchten Krebspatienten im Alter von 65-91 Jahren mind. ein Alternativpräparat einnahmen. Fatalerweise setzten besonders Patienten in frühen, noch gut kurativ behandelbaren Krebsstadien auf solche Arzneien (29\% in Stadium I-II, $15 \%$ in Stadium III-IV; Odds Ratio 2,05; 95\%-Konfidenzintervall: $1,21-3,49)$.

Vor allem pflanzliche Mittel lösten Wechselwirkungen aus, an 7,5\% der Interaktionen waren sie beteiligt. Am häufigsten wurden GlukosaminPräparate (19\%), Leinsamenöl (7\%) und Coenzym Q10 (6\%) eingenommen, aber auch „HochrisikoPräparate“ wie etwa Knoblauchpillen (3,5\%) oder Ginkgo-Zubereitungen (1\%). Sie könnten bekanntermaßen Stoffwechselprozesse negativ beeinflussen, die über Cytochrom-P-450 oder p-Glykoproteine vermittelt würden, so Ronald J. Maggiore, Chicago, IL/USA. Faktoren wie Alter und Krebsart beeinflussten das Einnahmeverhalten kaum. Das Problem sei den Autoren zufolge sogar noch gravierender, da etwa Vitaminpräparate in der Studie nicht berücksichtigt worden waren.

\section{Elke Oberhofer}

Maggiore RJ et al. Use of complementary medications among older adults with cancer. Cancer. 2012 Feb 22. doi: 10.1002/ cncr.27427. [Epub ahead of print]

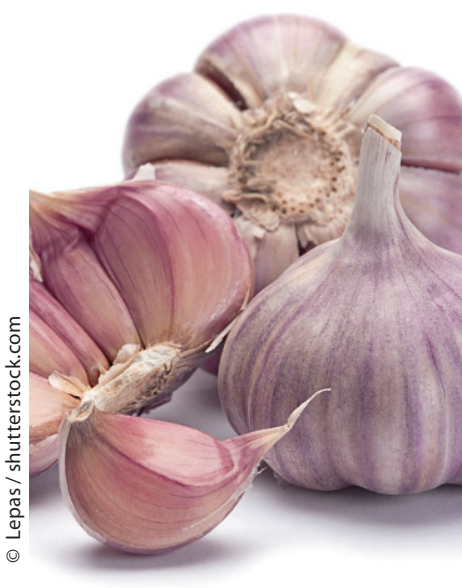

Nicht so unschuldig wie er aussieht: Krebspatienten sollten von Knoblauchpräparaten lieber die Finger lassen.

Laienverständliche Behandlungsleitlinien für Krebspatienten

Internetportal bringt Krebsinformationen auf den Punkt

Die Deutsche Gesellschaft für Hämatologie und Onkologie (DGHO) e.V. hat das Wissensportal www.mein-onkopedia.de erweitert. Das Onlineangebot bietet Patienten mit Krebs- und Bluterkrankungen eine verlässliche Quelle für alle Fragen rund um die Themen Untersuchung, Behandlung, Nebenwirkungen und Nachsorge. Aktuelle medizinische Leitlinien zu verschiedenen Blut- und Krebserkrankungen sind dort in eine allgemeinverständliche Sprache übersetzt. Ergänzt werden sie durch ein umfangreiches Glossar zu medizinischen Fachbegriffen mit direkter Verlinkung im Text, Hinweisen zu behandelnden Ärzten und Zentren vor Ort sowie Selbsthilfegruppen. Eine Kommentarfunktion am Ende jeder Patientenleitline ermöglicht dem Internetnutzer eine direkte Kontaktaufnahme zur Fachgesellschaft. Nähere Informationen darüber wie das Leitlinienportal funktioniert, bietet ein kurzer Informationsfilm unter: www.mein-onkopedia.de 